\title{
Deep-potential enabled multiscale simulation of gallium nitride devices on boron arsenide cooling substrates
}

Jing Wu ( $\nabla$ wujing_53@163.com )

Hunan University

E Zhou

Hunan University

An Huang

Hunan University

Hongbin Zhang

Institute of Materials Science, Technical University of Darmstadt https://orcid.org/0000-0002-10358861

\section{Ming Hu}

University of South Carolina

Guangzhao Qin

Hunan University

\section{Article}

Keywords:

Posted Date: January 25th, 2022

DOI: https://doi.org/10.21203/rs.3.rs-1256813/v1

License: (c) (i) This work is licensed under a Creative Commons Attribution 4.0 International License. Read Full License 


\section{Abstract}

High-efficient heat dissipation plays critical role for high-power-density electronics. Experimental synthesis of ultrahigh thermal conductivity boron arsenide (BAs, $1300 \mathrm{~W} \mathrm{~m}^{-1} \mathrm{~K}^{-1}$ ) cooling substrates into the wide-bandgap semiconductor of gallium nitride $(\mathrm{GaN})$ devices has been realized [Nature Electronics 4, 416-423 (2021)]. However, the lack of systematic analysis on the heat transfer across the BAs-GaN interface hampers the practical applications. In this study, by constructing the accurate and high-efficient machine learning interatomic potentials, we performed multiscale simulations of the BAs-GaN heterostructures. Ultrahigh interfacial thermal conductance (ITC) of $265 \mathrm{MW} \mathrm{m}^{-2} \mathrm{~K}^{-1}$ is achieved, which lies in the well-matched lattice vibrations of BAs and GaN. Moreover, the competition between grain size and boundary resistance was revealed with size increasing from $1 \mathrm{~nm}$ to $100 \mu \mathrm{m}$. Such deep-potential equipped multiscale simulations not only promote the practical applications of BAs cooling substrates in electronics, but also offer new approach for designing advanced thermal management systems.

\section{Introduction}

Thermal management is critical for electronic devices ${ }^{1-7}$, such as high-electron-mobility transistors (HEMTs), high-power density field-effect transistors (FETs), and radiofrequency (RF) devices, where high temperature degrades the performance of the system. However, in modern semiconductor industries ${ }^{8}$, the high hot-spot temperature in growing miniaturized electronic devices leads to a significant challenge for heat dissipation ${ }^{9,10}$. Thus, there is a strong impetus to search for high thermal conductivity (HTC) substrate materials, which can be integrated with hot-spot units for efficient heat dissipation. Therein the emerging interfacial thermal resistance (ITR) at the interface of heterostructure ${ }^{11-16}$ plays a crucial role, which may hamper the heat transfer as shown in Fig. 1(a). Consequently, HTC and low ITR are essential to heat dissipation.

Significant efforts have been employed to search, design, and investigate HTC materials for efficiently cooling down electronic systems ${ }^{17-28}$. Recently, some HTC compounds have been predicted based on first principles calculations. For instance, binary boron compounds and ternary boron compounds are reported $17,29,30$, both with thermal conductivity $(\kappa)$ higher than $500 \mathrm{~W} \mathrm{~m}^{-1} \mathrm{~K}^{-1}$. Especially, a remarkably high $\kappa$ of $1300 \mathrm{~W} \mathrm{~m}^{-1} \mathrm{~K}^{-1}$ for boron arsenide (BAs) has been confirmed by both theoretical calculations $\mathrm{s}^{31,32}$ and experimental measurements ${ }^{25-27}$.

Commonly, the high-power density electronic devices of HEMTs are composed of gallium nitride $(\mathrm{GaN})$, silicon, and silicon carbide substrates, where the performance bottleneck generally exists due to the low thermal conductivity. Recently, Kang. et al. ${ }^{33}$ successfully fabricated GaN devices on BAs cooling substrates in experiments ${ }^{34}$. Outstanding cooling efficiency compared with diamond-supported devices was observed, which is benefiting from the low ITR in addition to the ultrahigh $k$ of BAs. In fact, boundaries and interfaces are inevitable for the practical applications of cooling substrates in microelectronics. The interfacial thermal transport across $\mathrm{GaN}$ and the neighboring BAs is important for a 
high-performance thermal management, especially for the near-junction region. The fundamental understanding on the heat transfer across the BAs-GaN interface can offer insight for the design of advanced thermal management systems, which demands for a systematic study of the ITR.

Molecular dynamics (MD) simulations have been widely employed to study the thermal transport properties of interfaces in heterostructures ${ }^{11,35,36}$. Although it is robust and "automatic" to consider the effect of surface and interface, one apparent disadvantage of MD simulations is that it largely relies on an accurate interatomic potential. Unfortunately, the interatomic potentials of BAs are lacking up to now, which limits a detailed theoretical analysis on the interfacial thermal transport properties and thus hampers the potential applications of BAs as a competing cooling substrate for microelectronics. Recently, it has been demonstrated that machine learning (ML) techniques can be utilized to construct accurate interatomic potentials ${ }^{37-39}$, where artificial neural networks (ANN) and neural network potentials (NNPs) are the most widely used methods ${ }^{40,41}$. Correspondingly, the resulting multiscale simulations based on NNPs combine the advantages of the accuracy of $a b$ initio calculations and the efficiency of analytically parameterized potentials. Thus, by empowering the multiscale study of the thermal transport properties of heterostructure with first principles accuracy ${ }^{42,43}$, the NNPs land the foundation to promote in-depth understanding and predictive design of efficient heat dissipation for engineering microelectronic devices.

In this study, we constructed the NNPs for BAs, GaN, and their interfaces to systematically investigate the thermal transport properties of BAs-GaN heterostructure. The bulk thermal transport properties of $\mathrm{BAs}$ and $\mathrm{GaN}$ were firstly evaluated and validated with the existing reports. We then employed non-equilibrium MD (NEMD) and Monte Carlo (MC) simulations to evaluate and analyze the interface thermal transport properties of the BAs-GaN heterostructure. Finite element models (FEM) were further constructed to simulate BAs-GaN heterostructure at the size-scale from $1 \mathrm{~nm}$ to $100 \mathrm{~mm}$ to facilitate direct comparison with experimental observations.

\section{Results And Discussions}

\section{Neural network potential (NNP) training}

To investigate the heat transfer across the BAs-GaN interface as shown in Fig. 1, we firstly construct the NNPs for BAs, GaN, and the BAs-GaN heterostructure. The selection of configurations used in neural network (NN) training process is essential to construct reliable NNP models. More importantly, the interfacial structures in the BAs-GaN heterostructures can be complex. Therefore, the active learning strategy is utilized during the construction of NNPs, which shows high efficiency and low computation costs to collect relevant training configurations for pristine BAs, GaN, and BAs-GaN heterostructures. As shown in Fig. 2(a), the NNP training workflow comprises three steps (see the section of Methods for more details). With the increasing iterations of the $a b$ initio molecular dynamics (AIMD) simulations, we are able to collect diverse snapshots for the NNPs training. Finally, 2250, 2535, and 10350 training snapshots were collected for pristine BAs, GaN, and the BAs-GaN heterostructures, respectively. 
The quality of the resulting NNP models is verified by performing a comparison for energies and forces $\left(f_{x}, f_{y}, f_{z}\right)$ between density functional theory (DFT) calculations and NNPs predictions. The test configurations are composed of 2103, 2022 and 1990 snapshots for BAs, GaN and BAs-GaN heterostructure, respectively, which are not included in the configurations for previous training. From Fig. 2(d-i), it is obvious that NNPs are able to accurately reproduce the energies and forces obtained from DFT calculations. The force root mean square error (RMSE) are $0.05,0.15$, and $0.25 \mathrm{meV} / \AA$ for $\mathrm{BAs}, \mathrm{GaN}$, and BAs-GaN, respectively. The small RMSE also implies the high quality of the NNP models. With the NNP models, we calculate the phonon dispersions of $\mathrm{BAs}$ and $\mathrm{GaN}$, and the results coincide with those from explicit DFT calculations, as comparably shown in Fig. 2(b,c). Moreover, both BAs and GaN show obvious phonon gap, especially for BAs, which implies strong high order phonon scattering ${ }^{31}$.

\section{Thermal transport in BAs and $\mathrm{GaN}$}

With the well-trained NNP models, we can evaluate the lattice thermal conductivities ( $k$ ) of BAs and GaN. The finite displacement method was adopted to calculate the second- and third-order constants that are required to solve the Boltzmann transport equation (BTE) ${ }^{44-46}$. And the force calculations are performed using the Large-scale Atomic/Molecular Massively Parallel Simulator (LAMMPS) software package ${ }^{47}$ coupled with our trained NNPs. Fig. 3(b) shows the anisotropic $k$ of GaN, where the $k$ along the $a$ direction and $c$-direction are 357.08 and $296.26 \mathrm{~W} \mathrm{~m}^{-1} \mathrm{~K}^{-1}$, respectively. The results are in excellent agreement with previously reported DFT calculations ${ }^{48}$ of 354 and $287 \mathrm{~W} \mathrm{~m}^{-1} \mathrm{~K}^{-1}$, respectively. As for the BAs, when only considering three-phonon scatterings, the $K$ of BAs is $2198.38 \mathrm{~W} \mathrm{~m}^{-1} \mathrm{~K}^{-1}$ at room temperature [Fig. 3(a)], which is consistent with the reported value $\left(2241 \mathrm{~W} \mathrm{~m}^{-1} \mathrm{~K}^{-1}\right)$ in literature ${ }^{31}$. However, recent experiments and theoretical calculations demonstrated that BAs has strong four-phonon scatterings ${ }^{25,27,49}$. Different from the complex procedure of including four-phonon scatterings in $\mathrm{BTE}^{50}$, it is natural for MD simulations to consider the four-phonon scatterings and even higher order scattering.

To obtain the accurate $k$ of BAs from classical MD simulations, we performed the equilibrium MD (EMD) simulations based on the Green-Kubo (GK) method using the NNP models, where the thermal conductivity is evaluated based on the heat current autocorrelation function (HCACF):

$$
\left.K=\frac{V}{3 k_{B} T^{2}} \int_{0}^{\infty} U(0) \cdot J(t)\right\rangle d t
$$

1

where $V$ is the volume, $k_{B}$ is the Boltzmann constant, $T$ is the temperature, $J$ is the heat flux, and \langle\rangle represents the time average. The resulting HCACF is displayed in Fig. 3(c). It is shown that 800 ps EMD simulations can enable the generation of a converged $\kappa$ of BAs. For obtaining the $\operatorname{HCACF} U(0) \cdot J(t)\rangle$, the heat flux $J$ is calculated as follows: 


$$
J=\frac{1}{V} \sum_{i}\left(E_{i} \boldsymbol{v}_{i}-\boldsymbol{S}_{i} \cdot \boldsymbol{v}_{i}\right)
$$

where $E_{i}$ and $\boldsymbol{v}_{i}$ are the atomic potential energy and velocity, respectively. The atomic virial stress tensor $\boldsymbol{S}_{i}$ is defined as the outer product of relative atomic position $\boldsymbol{r}_{i}-\boldsymbol{r}_{j}$ and the derivative of local potential energy with respect to the neighboring atom position:

$$
\boldsymbol{S}_{i}=\sum_{i}\left(\boldsymbol{r}_{i}-\boldsymbol{r}_{j}\right) \otimes \frac{\partial E_{i}}{\partial\left(\boldsymbol{r}_{j}-\boldsymbol{r}_{i}\right)}
$$

To lower the simulation uncertainty and obtain reliable result, the $K$ is obtained by averaging five independent simulations as shown in Fig. 3(d). As summarized in Table 1 including the results from previous calculations $s^{31,32,51}$ and experiments ${ }^{25-27}$, the $\kappa$ of BAs obtained from MD simulations in this work $\left(1126.03 \mathrm{~W} \mathrm{~m}^{-1} \mathrm{~K}^{-1}\right)$ is well consistent with previous reports, especially compared with the BTE results involving four-phonon scattering and the experimental measurements. Note that it is for the first time the ultrahigh $k$ of BAs is verified from direct MD simulations of atomic motions. Besides, we also study other phonon related properties of BAs and $\mathrm{GaN}$, such as group velocity, phonon relaxation time, and the contribution of $\kappa$ from different phonon branches, which are provided in the Supplement materials.

Table 1 The comparison of $k(\mathrm{BAs})$ from MD simulations with previous reports from DFT calculations and experimental measurements. The 3-ph and 4-ph represent the phonon scattering involving three- and fourphonons, respectively.

\section{The interfacial thermal conductance (ITC) of the BAs-GaN heterostructures}

The interfacial heat transfer in the BAs-GaN heterostructures is further investigated by performing NEMD simulations. Fig. 4(a) shows a stable temperature profile of a steady state. There is an obvious temperature jump $(\Delta T)$ at the interfaces, implying a finite ITR between BAs and GaN. Thus, the ITC of the heterostructures is evaluated as $265 \mathrm{MW} \mathrm{m}^{-2} \mathrm{~K}^{-1}$ at $300 \mathrm{~K}$, which is in good agreement with the result ${ }^{33}$ measured by time-domain thermoreflectance (TDTR) $\left(250 \mathrm{MW} \mathrm{m}^{-2} \mathrm{~K}^{-1}\right)$ as collected in Fig. 4(b). Moreover, it can be seen that ITC gradually increases with respect to the increasing temperature. The underlying mechanism may lie in the more anharmonic features caused by the increasing temperature, which promotes more phonon transmission channels, and thus enhances the ITC ${ }^{11}$.

To fundamentally understand the behavior of ITR, the phonon density of states (PDOS) was explored for BAs and GaN. As shown in Fig. 4(c), there is an obvious band gap ranging from 10 to $16 \mathrm{THz}$, coincided with the phonon dispersions in Fig. 2(b,c). Moreover, the large overlap area of PDOS indicates the wellmatched lattice vibrations of BAs and GaN. Thus, the small ITR and the corresponding high ITC are 
achieved in the BAs-GaN heterostructures. Such conclusions would benefit the potential applications of BAs as a competing cooling substrate to diamond with the advantage of not needing epilayer ${ }^{7,52}$.

To further figure out the heat flux dependence on frequency, we use MC simulations to calculate the distribution of heat flux across the heterostructure interfaces, and the results are shown in Fig. 4(d). Apparently, there is a wide distribution range of heat flux for BAs, and the heat flux of GaN concentrates at low frequencies. Most importantly, the heat flux in the low frequency exhibits an excellent match across the interface, which explains the high ITC in the BAs-GaN heterostructures. Such behavior also implies that the low frequency phonons make more contributions to the ITC.

\section{Multiscale modeling from $1 \mathrm{~nm}$ to $100 \mathrm{~mm}$}

Based on the understanding of heat transfer process achieved from above DFT and MD simulations, the FEM can be employed to study the heterostructure models at a macroscopic scale. The combination of FEM and MD simulations is a promising approach to overcome the multiscale effect of grain sizes and the huge computational cost. Compared with MD and DFT simulations limited in the nanoscale, the macroscopic FEM can cohere with the practical applications and get the effective $k$ of BAs-GaN heterostructure in multiscale. Herein, a $1000 \mathrm{~nm}$ model is constructed with $50 \%$ BAs and $50 \% \mathrm{GaN}$ as shown in Fig. 5(a). To calculate the effective $\kappa$ of such a heterostructure model, one dimensional Fourier

law is applied as $K_{\text {eff }}=q \frac{L}{\Delta T}$, where $q$ is the heat flux across the model. The corresponding steady states are guaranteed when the value of the inward flux is equal to that of the outward flux. The $\Delta T$ is the temperature drop between the top side and bottom side, and Fig. 5(b) shows the resulting temperature profile.

From the FEM, Fig. 5(c) shows the ununiform heat flux distribution of $50 \%$ BAs and $50 \%$ GaN, respectively. It is observed that BAs dominates the majority of heat flux, which lies in that the thermal conductivity of BAs is significantly higher than that of GaN. Moreover, similar phenomena can also be seen from the two insets in Fig. 5(d), which consist of different percentages of BAs and GaN. To further understand the mechanism of grain size dependence of the effective $\kappa$, we perform a comparison study with grain size ranging from $1 \mathrm{~nm}$ to $100 \mu \mathrm{m}$. As shown in Fig. 5(d), there exists an important competition between the grain size and grain boundary. Firstly, when the grain size is below $10 \mathrm{~nm}$, the effective thermal conductivity decreases with increasing grain size. Such a behavior implies that the effect is negligible though the increasing size benefits phonon transport, which lies in the much smaller values than the representative MFP as indicated in Fig. $3(a, b)$. In fact, the increasing grain size results in more significant ITR, and the phonon scattering dominates the thermal transport. Consequently, the effective $\kappa$ decrease. Secondly, when the grain size ranges between $10 \mathrm{~nm}$ and $10 \mu \mathrm{m}$, BAs and GaN gradually activate more and more phonons to participate in the heat transfer with the increasing grain size. Thus, the increasing grain size leads to higher $\kappa$ to both BAs and $\mathrm{GaN}$, and the role of grain boundary becomes relatively less significant, leading to the significant increase of the effective $\kappa$. Finally, when the grain size is over $10 \mu \mathrm{m}$, the effective $\kappa$ tends to saturate. The reason lies in that the grain size 
greatly exceeds the phonon MFP of BAs and GaN [Fig. $3(a, b)]$, and the effect of grain boundary resistance largely weakens.

\section{Conclusion}

In summary, we have studied the thermal transport of GaN devices on BAs cooling substrates for highperformance thermal management. The state-of-the-art computational methods, i.e., ML, DFT, MD, and FEM, are employed to develop systematic multiscale simulations of the thermal transport properties together with quantitative evaluations and in-depth mechanistic evaluations. The comparison of DFT and MD simulations, and the excellent agreement with the reports from previous calculations and experiments show that the trained NNP models in this study are of high accuracy. And it is for the first time the ultrahigh thermal conductivity of BAs is verified from direct MD simulations of atomic motions. Ultrahigh ITC of $265 \mathrm{MW} \mathrm{m}^{-2} \mathrm{~K}^{-1}$ is obtained in the BAs-GaN heterostructures, which agrees well with experimental measurements, promising the conducive heat dissipation. Detailed analysis reveals that the underlying mechanism lies in the well-matched lattice vibrations of BAs and $\mathrm{GaN}$. Based on the understanding of heat transfer process achieved from nanoscale DFT and MD simulations, we successfully established the macroscopic FEM models of the BAs-GaN heterostructures and investigated the mechanism for the grain size dependent effective $k$ with the grain size ranging from $1 \mathrm{~nm}$ to $100 \mu \mathrm{m}$. Our results provide holistic and fundamental insights into the interfacial heat transfer of the BAs-GaN heterostructures, which is expected to promote the practical applications of BAs as a competing cooling substrate to diamond for electronic devices. Most importantly, we believe that our approach based on machine learning interatomic potential driven multiscale simulations can be applied to engineer and design sophisticated thermal management systems, and hence further promote the development of miniaturized electronic devices.

\section{Methods}

\section{DFT calculation}

The Vienna ab-initio simulation package (VASP), a widely employed ab initio plane wave electronic structure code for atomic scale materials modelling, was used to perform first-principles calculations with the Perdew-Burke-Ernzerhof functional of generalized gradient approximation ${ }^{53}$. For the DFT calculations, the kinetic energy cutoff was set as $1000 \mathrm{eV}$, a $10 \times 10 \times 6$ Monkhorst-Pack ${ }^{54} k$-point grid are employed for BAs and $\mathrm{GaN}$, respectively. For self-consistent field (SCF) calculations with convergence threshold of $10^{-6} \mathrm{eV}$, kinetic energy cutoff was set as $600 \mathrm{eV}$, and a $1 \times 1 \times 1$ Monkhorst-Pack k-point grid is employed.

\section{The construction of NNP models}

To collect diverse training data, active learning workflow is adopted ${ }^{55}$. To initialize the training data, $a b$ initio molecular dynamics (AIMD) was performed at $50 \mathrm{~K}$ using VASP, where the lattice constants of 
optimized structure was changed, and the scaling factors are $0.98,1$, and 1.02 , respectively. Besides, 0.03 $\AA$ A perturbations are introduced to some atoms for higher training accuracy. For the training, the smooth edition of the NNP is employed. A skip connection is adopted between two neighboring fitting layers. The cutoff is set to $7 \AA$. Four neural network models with Adam optimizer only combined with different parameter initializations, the embedding net with size of $\{30,60\}$, and the fitting net with size of $\{240,240$, 240 \} were trained. To the exploration step, when the force deviation of configurations is in the trust range $[0.05,0.2]$, it will be labeled. During the Labeling step, VASP was adopt to performance first-principles calculations. Finally, an accurate NNP model was trained with learning rate and training steps of 0.95 and 1000000 , respectively.

\section{MD simulation}

The LAMMPS software package was used to perform MD simulations. For EMD simulations, the structure was relaxed at canonical (NVT) ensembles with periodic boundary conditions for $0.1 \mathrm{~ns}$ with a time step of $1 \mathrm{fs}$. Then, the heat flux data can be started to collect. For NEMD simulations, first, the structure relaxed at isothermal-isobaric (NPT) ensemble. Then, the atomics of the two sides of the simulation system were fixed and 22 slabs were adopted to the remaining region. Heat source and heat sink is set to 320 and $280 \mathrm{~K}$, respectively. For the further relaxation with canonical (NVT) ensembles, the relaxation time is set as $1 \mathrm{~ns}$. Finally, the temperature of each slab and the heat flux across the system can be calculated.

\section{FEM simulation}

To construct polycrystalline models, ABAQUS/Standard with python script is used. The models are comprised of 2197 individual grains with Voronoi cells feature, which are randomly assigned with BAs or $\mathrm{GaN}$ attributes. Besides, the ITR between BAs and GaN grains are defined based on the NEMD results.

Assuming an equivalent average grain with cubic geometry ${ }^{56}$, we define the grain size as $\sqrt{\frac{L^{3}}{N}}$, where the

$N$ is the number of grain, and the $L$ is side length. Considering the $\mathrm{GaN}$ with anisotropic properties, we assign corresponding grain with random orientations. We apply two highly conductive sheets to construct heat source and cold source. For the initial load condition, we set the temperature of cold source as zero.

\section{Declarations}

\section{Acknowledgement}

We acknowledge the support of the National Natural Science Foundation of China (Grant Nos. 52006057) and the Fundamental Research Funds for the Central Universities (Grant Nos. 531119200237 and 541109010001). This work was also supported in part by the NSF (award number 2110033). The numerical calculations in this paper have been done on the supercomputing system of the National Supercomputing Center in Changsha. 


\section{Data availability}

The datasets (NNP models, LAMMPS input files, ShengBTE output files and FE models) in this study are available at https://github.com/WU2JING/H.

\section{References}

1. Moore, A. L. \& Shi, L. Emerging challenges and materials for thermal management of electronics. Mater. Today 17, 163-174 (2014).

2. Zheng, Q. et al. High Thermal Conductivity in Isotopically Enriched Cubic Boron Phosphide. Adv. Funct. Mater. 28, 1805116 (2018).

3. Samantaray, C. B. \& Singh, R. N. Review of synthesis and properties of cubic boron nitride (c-BN) thin films. Int. Mater. Rev. 50, 313-344 (2005).

4. Cui, Y., Qin, Z., Wu, H., Li, M. \& Hu, Y. Flexible thermal interface based on self-assembled boron arsenide for high-performance thermal management. Nat. Commun. 12, 1284 (2021).

5. Guo, X., Cheng, S., Cai, W., Zhang, Y. \& Zhang, X. A review of carbon-based thermal interface materials: Mechanism, thermal measurements and thermal properties. Mater. Des. 209, 109936 (2021).

6. Kang, J. S., Wu, H. \& Hu, Y. Thermal Properties and Phonon Spectral Characterization of Synthetic Boron Phosphide for High Thermal Conductivity Applications. Nano Lett. 17, 7507-7514 (2017).

7. Huang, X. \& Guo, Z. Thermal effect of epilayer on phonon transport of semiconducting heterostructure interfaces. Int. J. Heat Mass Transf. 178, 121613 (2021).

8. Ball, P. The more that microcircuits are shrunk, the Feeling hotter they get. Engineers are on the hunt for ways to cool the heat off computing. 3.

9. Malakoutian, M. et al. Record-Low Thermal Boundary Resistance between Diamond and GaN-on-SiC for Enabling Radiofrequency Device Cooling. ACS Appl. Mater. Interfaces acsami.1c13833 (2021) doi:10.1021/acsami.1c13833.

10. Cheng, Z., Mu, F., Yates, L., Suga, T. \& Graham, S. Interfacial Thermal Conductance across RoomTemperature-Bonded GaN/Diamond Interfaces for GaN-on-Diamond Devices. ACS Appl. Mater. Interfaces 12, 8376-8384 (2020).

11. Ren, K. et al. Remarkable Reduction of Interfacial Thermal Resistance in Nanophononic Heterostructures. Adv. Funct. Mater. 30, 2004003 (2020).

12. Yang, L., Wan, X., Ma, D., Jiang, Y. \& Yang, N. Maximization and minimization of interfacial thermal conductance by modulating the mass distribution of the interlayer. Phys. Rev. B 103, 155305 (2021).

13. Xiong, Y. et al. Ultralow thermal conductance of the van der Waals interface between organic nanoribbons. Mater. Today Phys. 11, 100139 (2019).

14. Ren, W. et al. The Impact of Interlayer Rotation on Thermal Transport Across Graphene/Hexagonal Boron Nitride van der Waals Heterostructure. Nano Lett. 21, 2634-2641 (2021). 
15. Wu, Y.-J., Fang, L. \& Xu, Y. Predicting interfacial thermal resistance by machine learning. Npj Comput. Mater. 5, 56 (2019).

16. Li, T., Tang, Z., Huang, Z. \& Yu, J. Substrate effects on the thermal performance of in-plane graphene/hexagonal boron nitride heterostructures. Carbon 130, 396-400 (2018).

17. Mortazavi, B., Novikov, I. S. \& Shapeev, A. V. A machine-learning-based investigation on the mechanical/failure response and thermal conductivity of semiconducting BC2N monolayers. Carbon 188, 431-441 (2022).

18. Pang, Z., Gu, X., Wei, Y., Yang, R. \& Dresselhaus, M. S. Bottom-up Design of Three-Dimensional Carbon-Honeycomb with Superb Specific Strength and High Thermal Conductivity. Nano Lett. 17, 179-185 (2017).

19. Ouyang, W., Qin, H., Urbakh, M. \& Hod, O. Controllable Thermal Conductivity in Twisted Homogeneous Interfaces of Graphene and Hexagonal Boron Nitride. Nano Lett. 20, 7513-7518 (2020).

20. Kim, S. E. et al. Extremely anisotropic van der Waals thermal conductors. Nature 597, 660-665 (2021).

21. Tian, F. \& Ren, Z. High Thermal Conductivity in Boron Arsenide: From Prediction to Reality. Angew. Chem. 131, 5882-5889 (2019).

22. Hu, Y. et al. High thermal conductivity in covalently bonded bi-layer honeycomb boron arsenide. Mater. Today Phys. 17, 100346 (2021).

23. Cai, Q. et al. High thermal conductivity of high-quality monolayer boron nitride and its thermal expansion. Sci. Adv. 5, eaav0129 (2019).

24. Rodriguez, A., Lam, S. \& Hu, M. Thermodynamic and Transport Properties of LiF and FLiBe Molten Salts with Deep Learning Potentials. ACS Appl. Mater. Interfaces acsami.1c17942 (2021) doi:10.1021/acsami.1c17942.

25. Tian, F. et al. Unusual high thermal conductivity in boron arsenide bulk crystals. Science 361, 582585 (2018).

26. Kang, J. S., Li, M., Wu, H., Nguyen, H. \& Hu, Y. Experimental observation of high thermal conductivity in boron arsenide. Science 361, 575-578 (2018).

27. Li, S. et al. High thermal conductivity in cubic boron arsenide crystals. Science 361, 579-581 (2018).

28. Graham, S. \& Choi, S. Integrating boron arsenide into power devices. Nat. Electron. 4, 380-381 (2021).

29. Lin, C., Zhang, X. \& Rao, Z. Theoretical prediction of thermal transport in BC $2 \mathrm{~N}$ monolayer. Nano Energy 38, 249-256 (2017).

30. Wu, H., Fan, H. \& Hu, Y. Ab initio determination of ultrahigh thermal conductivity in ternary compounds. Phys. Rev. B 103, L041203 (2021).

31. Feng, T., Lindsay, L. \& Ruan, X. Four-phonon scattering significantly reduces intrinsic thermal conductivity of solids. Phys. Rev. B 96, 161201 (2017). 
32. Liu, Z., Yang, X., Zhang, B. \& Li, W. High Thermal Conductivity of Wurtzite Boron Arsenide Predicted by Including Four-Phonon Scattering with Machine Learning Potential. ACS Appl. Mater. Interfaces 13, 53409-53415 (2021).

33. Kang, J. S. et al. Integration of boron arsenide cooling substrates into gallium nitride devices. Nat. Electron. 4, 416-423 (2021).

34. Yang, S. et al. Reduced thermal boundary conductance in GaN-based electronic devices introduced by metal bonding layer. Nano Res. 14, 3616-3620 (2021).

35. Liu, X., Zhang, G. \& Zhang, Y.-W. Topological Defects at the Graphene/ $h$-BN interface Abnormally Enhance Its Thermal Conductance. Nano Lett. 16, 4954-4959 (2016).

36. Liu, F. et al. Enhancement of thermal energy transport across the graphene/h-BN heterostructure interface. Nanoscale 11, 4067-4072 (2019).

37. Friederich, P., Häse, F., Proppe, J. \& Aspuru-Guzik, A. Machine-learned potentials for next-generation matter simulations. Nat. Mater. 20, 750-761 (2021).

38. Qian, X. \& Yang, R. Machine learning for predicting thermal transport properties of solids. Mater. Sci. Eng. R Rep. 146, 100642 (2021).

39. Rodriguez, A., Liu, Y. \& Hu, M. Spatial density neural network force fields with first-principles level accuracy and application to thermal transport. Phys. Rev. B 102, 035203 (2020).

40. Zhang, L., Wang, H., Car, R. \& E, W. Phase Diagram of a Deep Potential Water Model. Phys. Rev. Lett. 126, 236001 (2021).

41. Niu, H., Bonati, L., Piaggi, P. M. \& Parrinello, M. Ab initio phase diagram and nucleation of gallium. Nat. Commun. 11, 2654 (2020).

42. Zhang, L., Han, J., Wang, H., Car, R. \& E, W. Deep Potential Molecular Dynamics: A Scalable Model with the Accuracy of Quantum Mechanics. Phys. Rev. Lett. 120, 143001 (2018).

43. Zeng, J., Cao, L., Xu, M., Zhu, T. \& Zhang, J. Z. Complex Reaction Processes in Combustion Unraveled by Neural Network Based Molecular Dynamics Simulation. 24.

44. Li, W., Carrete, J., Katcho, N. A. \& Mingo, N. ShengBTE: A solver of the Boltzmann transport equation for phonons. Comput. Phys. Commun. 12 (2014).

45. Li, W., Lindsay, L., Broido, D. A., Stewart, D. A. \& Mingo, N. Thermal conductivity of bulk and nanowire Mg 2 Si x Sn $1-x$ alloys from first principles. Phys. Rev. B 86, 174307 (2012).

46. Togo, A. \& Tanaka, I. First principles phonon calculations in materials science. Scr. Mater. 108, 1-5 (2015).

47. Plimpton, S. Fast Parallel Algorithms for Short-Range Molecular Dynamics. 42.

48. Qin, Z., Qin, G., Zuo, X., Xiong, Z. \& Hu, M. Orbitally driven low thermal conductivity of monolayer gallium nitride $(\mathrm{GaN})$ with planar honeycomb structure: a comparative study. Nanoscale 9 , 42954309 (2017).

49. Dames, C. Ultrahigh thermal conductivity confirmed in boron arsenide. Science 361, 549-550 (2018). 
50. Han, Z., Yang, X., Li, W., Feng, T. \& Ruan, X. FourPhonon: An extension module to ShengBTE for computing four-phonon scattering rates and thermal conductivity. Comput. Phys. Commun. 270, 108179 (2022).

51. Yang, X., Feng, T., Li, J. \& Ruan, X. Stronger role of four-phonon scattering than three-phonon scattering in thermal conductivity of III-V semiconductors at room temperature. Phys. Rev. B 100, 245203 (2019).

52. Liang, J. et al. Fabrication of GaN/Diamond Heterointerface and Interfacial Chemical Bonding State for Highly Efficient Device Design. Adv. Mater. 33, 2104564 (2021).

53. Perdew, J. P., Burke, K. \& Ernzerhof, M. Generalized Gradient Approximation Made Simple. Phys. Rev. Lett. 77, 3865-3868 (1996).

54. Monkhorst, H. J. \& Pack, J. D. Special points for Brillouin-zone integrations. Phys. Rev. B 13, 51885192 (1976).

55. Zhang, Y. et al. DP-GEN: A concurrent learning platform for the generation of reliable deep learning based potential energy models. Comput. Phys. Commun. 253, 107206 (2020).

56. Mortazavi, B., Pötschke, M. \& Cuniberti, G. Multiscale modeling of thermal conductivity of polycrystalline graphene sheets. Nanoscale 6, 3344-3352 (2014).

\section{Table 1}

Table 1 is available in the Supplementary Files section.

\section{Figures}

\section{Figure 1}

Thermal management employing the boron arsenide (BAs) cooling substrates for gallium nitride (GaN) devices. (a) The schematic of BAs supporting GaN HEMTs and the distribution of hot-spot temperature. (b-c) The crystal structures of unit cell of BAs and GaN, respectively. (d) The phonon transmission and reflection models at the heterostructure interface. (e) The steady state of temperature profile for BAs-GaN heterostructure and the formula of interfacial thermal resistance (ITR), where $Q$ and $\Delta T$ are the heat flux and temperature drop, respectively.

\section{Figure 2}

The active learning workflow and the accuracy verification of the constructed NNP. (a) The integration of three steps of the training of NNP models, exploration of new configuration, and calculation of energies 
and forces at DFT level. Besides, the structure of the NNP models is also presented. (b-c) The comparison of phonon dispersion of (b) BAs and (c) GaN between NNP and DFT. (d-i) The comparison of energies and forces of BAs, GaN, BAs-GaN heterostructure from NNP predictions and DFT calculations, where color indicates the number density of points.

\section{Figure 3}

The thermal conductivity ( $k$ ) of BAs and GaN. (a-b) Comparison of the accumulative $k$ with respect to phonon mean free path (MFP) for (a) BAs at different temperatures and (b) GaN with anisotropy, respectively. The insets are electron localization function (ELF). (c-d) The converging (c) normalized HCACF and the (d) corresponding $k$ at $300 \mathrm{~K}$ for BAs as a function of simulation time.

\section{Figure 4}

The analysis of thermal transport in BAs-GaN heterostructure from MD and MC simulations. (a) The temperature distribution in the BAs-GaN heterostructure in steady state. Inset is the model for NEMD simulations. (b) The ITC with respect to temperature, in comparison with experimental measurements. (c) The comparison of phonon DOS between BAs and GaN. (d) The spectral heat flux in BAs-GaN heterostructure, where the dash line indicates the interface and the color indicates the density of flux.

\section{Figure 5}

The FEM models and the effective $\kappa$ for different percentages of BAs and GaN. (a) The FEM model with $50 \%$ BAs and $50 \% \mathrm{GaN}$, and the multiscale Schematic. (b-c) The temperature profile and the distribution of heat flux at the typical size of $1 \mathrm{~mm}$. (d) The effective $k$ of different proportion BAs and GaN models with respect to the grain size. The distribution of heat flux of $40 \% \mathrm{BAs}-60 \% \mathrm{GaN}$, and $60 \% \mathrm{BAs}-40 \%$ $\mathrm{GaN}$ are shown as inset. The colored ellipse marks the turning point of the effective $\kappa$ with the increasing grain size.

\section{Supplementary Files}

This is a list of supplementary files associated with this preprint. Click to download.

- SupplementaryMaterials.docx

- floatimage3.png 